\title{
Über Potenzreihen, deren Koeffizienten zahlentheoretische Funktionen sind.
}

Von

G: Szegó in Budapest.

Herr G. Pólya hat mir im Jahre 1915 folgende Vermutung brieflich mitgeteilt:

Hat die Potenzreihe

$$
f(z)=a_{0}+a_{1} z+\ldots+a_{n} z^{n}+\ldots
$$

lauter ganzzahlige Koeffizienten und den Konvergenzradius 1, so stellt sie entweder eine rationale Funktion dar oder sie ist über den Einheitskress hinaus nicht fortsetzbar, d. h. sie hat den Einheitskreis zur natïrlichen Grenze ${ }^{1}$ ).

Die Frage, ob diese Vermutung zutrifft oder nicht, ist bis heute unentschieden. Die weitgehendsten Resultate, welche in dieser Hinsicht bisher erreicht wurden, sind folgende:

Ein Satz von Herrn G. Pólya: Es sei $R>1$ und die Funktion $f(z)$ im Kreise $\mid z \leqq R$ eindeutig und mit Ausnahme einer endlichen Anzahl von singulären Stellen regulär. Wenn $a_{n}$ für alle $n$ ganz ist, so ist $f(z)$ eine rationale Funktion ${ }^{2}$ ).

Ein Satz von Herrn F. Hausdorff: Die Potenzreihen mit lauter ganzzahligen Koeffizienten und dem Konvergenzradius 1, die über den Einheitskreis hinaus fortsetzbar sind, bilden eine abzählbare $M$ Nenge $^{3}$ ).

1) Man vgl. G. Pólya, tber Potenzreihen mit ganzzahligen Koeffizienten Mathematische Annalen 77 (1916), S. 497-513], S. 510.

9) A. a. O. S. 497. Vgl. noeh die Arbeiten von R. Jentzsch, G. Pólya, F. Carlson, eämtliche unter dem Titel Ober Potenzreihen mit endlich vielen verschiedenen Koeffizienten in den Mathematischen Annalen bzw. 78, S. 276-285, 78 , S. $286-293,79$, S. $237-245$.

3) F. Hausdorff, Zur Verteilung der fortsetzbaren Potenzreiben, Mathematische Zeitschrift, 4, S. 98-103. 
Der Zweck dieser Arbeit ist die Nichtfortsetzbarkeit über den Einheitskreis hinaus für einige Potenzreihen, deren Koeffizienten gewisse wohlbekannte zahlentheoretische Funktionen von $n$ sind, nachzuweisen. Dies wird erreicht durch eine einheitliche Methode, die sich einerseits auf einen Satz über Dirichletsche Reihen, andererseits auf gewisse Umformungen dieser Reihen stützt.

Einige der hier untersuchten Reihen sind bereits von anderen Autoren behandelt worden ${ }^{4}$ ). Für gewisse andere unter ihnen folgt ferner die Eigenschaft der Nichtfortsetzbarkeit aus allgemeinen Kriterien ${ }^{5}$ ). Doch glaube ich, daß die hier dargelegte Methode ein Interesse verdient, nicht bloß darum, weil sie die Nichtfortsetzbarkeit, welche für die meisten dieser Reihen unbekannt war, liefert, sondern noch mehr darum, weil es immer merkwürdig ist, wenn zahlentheoretische Betrachtungen auf die Lösung funktionentheoretischer Fragen führen.

\section{Inhalt.}

\$ 1. Hilfssätze aus der analytischen Zạhlentheorie.

\$ 2. Umformung Dirichletscher Reihen.

$\S$ 3. Darstellung der Methode.

$\S$ 4. $\sum_{n=1}^{\infty} z^{p_{n}}$.

$\S 5 . \sum_{n=1}^{\infty} 1(n) z^{n}$.

4) Die Reihen in den $\$ \$ 6$ und 7 sind als Lambertsche Reihen darzustellen, ihre Nichtfortsetzbarkeit folgt aus gewissen allgemeinen Sätzen über Lambert Reihen. Man vgl. J. Franel, Sur la théorie des séries Mathematisehe Annalen 52 (1899), S. $529-549$, S. 548; ferner K. Knopp, Vber Lambertsehe Reihen Journal für die reine und angewandte Mathematik, 142 (1913), S. 283-315), S. $313,314$.

Uber die Potenzreihen in $\$ 9$ hat Fatou bewiesen, dab für sie $z=1$ süngulärer Punkt ist. P. Fatou, Séries trigonométriques et séries de Taylor Acta Mathematica, 30 (1906), S. 335-400), S. 392.

3) Es gilt der Fabrysehe Satz:

Die Potenzreike

$$
\sum_{n=1}^{\infty} a_{n,} z^{m_{\nu}}
$$

hat den Einheitskreis zur natürlichen Grenze, wenn

$$
\varlimsup_{r=\infty}^{m_{y}} \sqrt{a_{m_{v}}}=1 \text { and } \lim _{v=\infty} \frac{m_{x}}{v}=\infty
$$

ist. Dieser Satz führt bei den Reihen in $\$ \$ 4,5$ zum Ziele 


$$
\begin{aligned}
& \text { §. } \sum_{n=1}^{\infty} \circ(n) z^{n} \text { und } \sum_{n=1}^{\infty} \tau(n) z^{n} \text {. } \\
& \text { § 7. } \sum_{n=1}^{\infty} \varphi(n) z^{n} \text {. } \\
& \text { \& } 8 . \sum_{n=1}^{\infty} 2^{n(n)} z^{n k} \text {. } \\
& \text { §. } \sum_{n=1}^{\infty} \mu(n) \dot{z} \text {. } \\
& \S 10 . \sum_{n=1}^{\infty}[\mu(n)]^{2} z^{n} \\
& \S 11 . \sum_{n=1}^{\infty} 2^{n(n)} \lambda(n) z^{n}
\end{aligned}
$$

$\S 1$.

\section{Hilfssätze aus der analytischen Zahlentheorie ${ }^{6}$ ).}

Im folgenden bezeichne ich mit $k$ immer eine ungerade Primzahl und mit $g$ eine primitive Wurzel modulo $k$, d. h. eine solche zu $k$ teilerfremde Zahl, für welche

$$
1, g, g^{2}, \ldots, g^{k-2}
$$

inkongruent sind modulo $k$. Dann gehört zu jeder Zahl $m$, für welche $(m, k)=1$ ist, eine ganz bestimmte $\nu$ mit der Eigenschaft

$$
m=g^{r}(\bmod k) \quad(0 \leqq \nu \leqq k-2) .
$$

Ist $m=-m^{\prime}(\bmod k)$, so gehört zu $m$ und $m^{\prime}$ dieselbe $\nu$. Ich setze $v=j_{m}$.

Ich definiere jetzt gewisse zahlentheoretische Funktionen, die sog. Charaktere auf folgende Weise: Es sei

$$
Q=e^{\frac{2 \pi}{k-1} i} \text { und } \chi_{c e}(n)=Q^{\left(i j_{n}\right.}
$$

wenn $n$ nicht durch $k$ teillbar ist. Andernfalls sei $\chi_{\alpha}(n)=0$ ( $\alpha$ ist ganz).

Diese Funktionen besitzen folgende elementare Eigenschaften:

$$
\begin{aligned}
& \text { a) } \chi_{\alpha x}(n)=\chi_{\alpha^{\prime}}(n) \text { für } c \equiv a^{\prime}(\bmod k-1) \text {. } \\
& \text { b) } \chi_{\alpha}(n)=\chi_{\alpha}\left(n^{\prime}\right) \text { für } n \equiv n^{\prime}(\bmod k) \text {. } \\
& \text { c) } \chi_{\alpha x+\alpha^{\prime}}(n)=\chi_{\alpha}(n) \chi_{a^{\prime}}(n) .
\end{aligned}
$$

9) Man vgl. E. Landau, Handbuch der Lchre von der Verteilung der Primzahlen, 2 Bände, Leipzig und Berlin (B. G. Teubner) 1909, 1, S. 391-417 und S. 475-498. Ioh zitiere dieses Buch im folgenden mit $\mathrm{H}$. 
Potenzreiben, dẹen Keeffizienten zahlentheoretisohe Funktionen sind.

d) $\chi_{\alpha}\left(n n^{\prime}\right)=\chi_{\alpha z}(n) \chi_{\alpha}\left(n^{\prime}\right)$.

e) $\sum_{n=0}^{k-1} x_{\alpha}(n)=\left\{\begin{array}{ccc}k-1 & \text { für } & \text { c }=0, \\ 0 & \text { für } & 1 \leqq \alpha \leqq k-2 .\end{array}\right.$

f) $\sum_{\alpha=0}^{k-2} \chi_{\alpha}(n)=\left\{\begin{array}{cl}k-1 & \text { für } n \equiv 1(\bmod k), \\ 0 & \text { in allen anderen Fällen. }\end{array}\right.$

Ich definiere ferner folgende analytische Funktionen:

$$
\begin{aligned}
& L_{\alpha}(s)=L_{a}(s)=\sum_{n=:}^{\infty} \frac{\chi_{\alpha}(n)}{n^{s}}=\prod_{p} \frac{1}{1-\frac{\chi_{\alpha c}(p)}{p^{s}}} \\
&\left(c=0,1, \ldots, k-2 ; c^{\prime} \equiv a, \bmod k-1\right)
\end{aligned}
$$

wo $s=\sigma+i t$ eine komplexe Veränderliche bezeichnet und $p$ alle Primzahlen durchläuft. Diese Reihen sind sämtlich absolut konvergent, wenn $\sigma=\Re s>1$ ist. Ich zitiere die Hilfssätze: Die Funktion

$$
L_{0}(s)=\left(1-\frac{1}{k^{s}}\right) \zeta(s)
$$

wo $\zeta(s)$ die Riemannsche Zetafunktion bezeichnet, ist eine meromorphe Funktion, welche im Endlichen die einzige Singularität $s=1$ hat. Die ganze transzendente Funktion $(s-1) \zeta(s)$ verschwindet für $s=-2$, $-4,-6, \ldots$ und ihre übrigen Wurzeln liegen alle im Streifen

$$
0<\sigma<1 \text {. }
$$

Ist $1 \leqq \alpha \leqq k-2$, so ist $L_{\alpha}(s)$ eine ganze transzendente Funktion von der folgenden Form:

$$
L_{\alpha}(s)=\left(\frac{\pi}{k}\right)^{\frac{s+\delta_{\alpha}}{q}} \frac{1}{\Gamma\left(\frac{s+\delta_{\alpha}}{2}\right)^{\xi_{\alpha}}(s),}
$$

wo $\delta_{\alpha}=0$ oder 1 , je nachdem $a$ gerade oder ungerade ist, $\Gamma(s)$ bezeichnet die Euler sche Gammafunktion und $\xi_{\alpha}(s)$ die folgenden Eigenschaften besitzt:

a) Sie 'ist eine ganze transzendente Funktion.

b) Ihre Wurzeln liegen im Streifen

$$
0<\sigma<1 \text {. }
$$

c) Sie genügt der Funktionalgleichung

$$
\xi_{\alpha}(s)=\varepsilon_{\alpha} \xi_{k-1-\alpha}(1-s) \quad(1 \leqq \varepsilon \leqq k-2
$$

wo $\varepsilon_{\alpha}$ eine nar von $\alpha$ und $k$ abhängende Konstante bezeichnet mit dem absoluten Betrage 1 . 


\section{Umformung Dirichletscher Reihen.}

Es sei $\varepsilon \neq 1$ eine $k$-te Einheitswarzel und

$$
g(s)=\sum_{n=1}^{\infty} \frac{a_{n} \varepsilon^{n}}{n^{s}}
$$

eine Dirichletsche Reihe, die für genügend große $\sigma$ absolut konvergiert. Ich setze

dann ist

$$
A(s)=\sum_{n=1}^{\infty} \frac{a_{n}}{n^{s}}, \quad A_{r}(s)=\sum_{n=r(\bmod k)} \frac{a_{n}}{n^{s}} \quad(0 \leqq r \leqq k-1)
$$

$$
g(s)=\sum_{r=0}^{k-1} \varepsilon_{n=r(\bmod k j}^{r} \frac{a_{n}}{n^{s}}=\sum_{r=0}^{k-1} \varepsilon^{r} A_{r}(s) .
$$

Ferner definiere ich folgende analytische Funktionen:

(3) $M_{a}(s)=\sum_{r=0}^{k-1} z_{\alpha}(r) A_{r}(s)=\sum_{r=0}^{k-1} \sum_{n=\tau(\bmod k)} \frac{a_{n} x_{a}(n)}{n^{s}}=\sum_{n=1}^{\infty} \frac{a_{n} \chi_{a}(n)}{n^{s}}$

Nun betrachte ich das lineare System

$$
(c=0,1, \ldots, k-2) \text {. }
$$

$$
\begin{gathered}
x_{0} x_{0}(1)+x_{1} x_{1}(1)+\ldots+x_{k-2} x_{k-9}(1)=\varepsilon \\
x_{0} x_{0}(2)+x_{1} x_{1}(2)+\ldots+x_{k-3} x_{k-2}(2)=\varepsilon^{2} \\
\ldots
\end{gathered}
$$

$$
x_{0} \chi_{0}(k-1)+x_{1} \chi_{1}(k-1)+\ldots+x_{k-2} \chi_{k-2}(k-1)=\varepsilon^{k-1} .
$$

Die Determinante

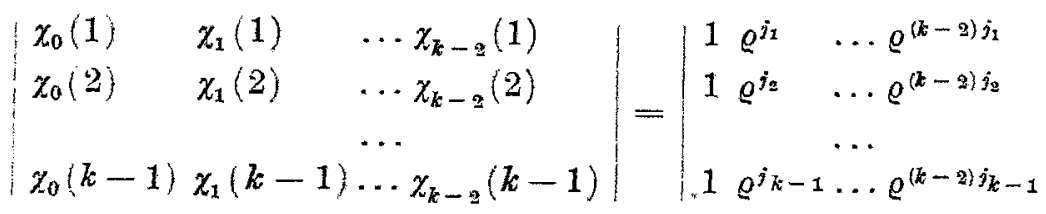

ist von 0 verschieden, $\mathrm{da} \varrho^{j_{\alpha}} \neq \varrho^{i_{\beta}}$ ist für $\alpha \neq \beta$ und $1 \leqq \alpha, \beta \leqq k-1$. Das System gestattet somit eine "ganz bestimmte Lösung und die Zahlen $x_{0}, x_{1}, \ldots, x_{k-2}$ hängen nur von $k$ und $\varepsilon a b$.

Durch Addieren der obigen Gleichungen ergibt sich

d. $\mathbf{h}$.

$$
x_{0}(k-1)=\varepsilon+\varepsilon^{2}+\ldots+\varepsilon^{k-1}=-1,
$$

$$
x_{0}=\frac{1}{1-k} \text {. }
$$

Ich bemerke ferner, daß die Zahlen $x_{1}, x_{3}, \ldots, x_{k-3}$ nicht sämtlich 
verschwinden. Ist nämlich $j_{r}=\frac{k-1}{2}(2 \leqq r \leqq k-1)$, so hat man nach der ersten und $r$-ten Gleichung

$$
\begin{aligned}
& x_{0}+x_{1}+\ldots+x_{k-2}=\varepsilon \\
& x_{0}-x_{1}+\ldots-x_{k-2}=\varepsilon^{r}
\end{aligned}
$$

und $\varepsilon$ ist verschieden von $\varepsilon^{r}$.

Es folgt num

$$
\begin{aligned}
\sum_{u=0}^{k-2} x_{\alpha} M_{\alpha}(s) & =\sum_{a=0}^{k-2} \sum_{r=0}^{k-1} x_{\alpha} z_{\alpha}(r) A_{r}(s)=\sum_{r=0}^{k-1} \sum_{\alpha=1}^{k-2} x_{\alpha} \chi_{\alpha}(r) A_{r}(s) \\
& =\sum_{r=1}^{k-1} \varepsilon^{r} A_{r}(s)=g(s)-A_{0}(s)
\end{aligned}
$$

d. h.

$$
\begin{aligned}
g(s) & =A_{0}(s)+\sum_{\alpha=0}^{k-2} x_{\alpha} M_{\alpha}(s) \\
& =A(s)+\left(x_{0}-1\right) M_{0}(s)+\sum_{\alpha=1}^{k-2} x_{\alpha} M_{\alpha}(s) .
\end{aligned}
$$

Diese Umformung von $g(s)$ gilt vorlänfig nur für genug große $\sigma$; sie git aber nach dem Prinzip der analytischen Fortsetzung in der $s$ - Kabene überall, wo die Funktionen $A(s), M_{0}(s), M_{1}(s), \ldots, M_{k-9}(s)$ sämtlich regulär sind.

Es sei jetzt

\section{$\S 3$.}

\section{Darstellung der Methode.}

$$
f(z)=a_{1} z+a_{2} z^{2}+\ldots+a_{n} z^{n}+\ldots
$$

eine Potenzreihe mit dem Konvergenzradius 1. Thre Nichtfortsetzbarkeit über den Einheitskreis hinaus wixd offenbar bewiesen, wenn ich die Existenz einer überall dichten Menge von singulären Stellen am Rande des Linheitskreises nachweise. Für die Reihen, die hier behandelt werden, zeige ich insbesondere, daß die $k$-ten (von 1 verschiedenen) Einheitswurzeln singuläre Stellen sind, wo $k$ unendlich viele Primzahlen durchläuft.

Jis gilt nämlich folgender Satz:

Es habe die Dirichletsche Reihe

$$
\gamma(s)=\sum_{n=1}^{\infty} \frac{\alpha_{n}}{n s}
$$

eine endliche Konvergenzaỏszisse; folglich ist

$$
q(z)=\sum_{x=1}^{\infty} a_{n} z^{n}
$$


regulär für $|z|<1$. Ist $\varphi(z)$ regulär auch für $z=1$, so ist $\gamma(s)$ eine ganze transzendente Funktion ${ }^{7}$ ).

- Es sei also von nun an $k$ eine feste ungerade Primzahl und $\varepsilon$ eine ebenfalls feste, von 1 verschiedene $k$-te Einheitswurzel; ist $f(z)$ für $z=\varepsilon$ regulär, so ist

$$
g(s)=\sum_{n=1}^{\infty} \frac{a_{n} \varepsilon^{n}}{n^{3}}
$$

eine ganze transzendente Funktion, vorausgesetzt, daß $a_{n}=O\left(n^{r}\right)$ ist. In den Fällen, die hier behandelt werden, kostet die Berechnung der Funktionen (3) (d. h. ihre Zurückführung auf $L_{\alpha}(s)$ und $\zeta(s)$ ) keine besondere Mühe. Ich zeige nun mit Hilfe der Formel $\left(5^{\prime}\right)$, daß die Funktion $g(s)$ an gewissen Stellen der $s$-Ebene Pole besitzt; daraus folgt aber die Unmöglichkeit der Annahme, daß $f(z)$ für $z=\varepsilon$ regulär ist.

Die Hauptschwierigkeit bei diesem Beweise besteht darin, daß mehrere Glieder der Summe ( $5^{\prime}$ ) gleichzeitig singulär werden; die Aufgabe reduziert sich somit darauf, das Nichtverschwinden von den Residuen oder, allgemeiner, von gewissen Gliedern des singulären Teils der Laurentschen Entwicklung nachzuweisen.

Ich behalte durchwegs die Bezeichnungen (1), (2), (3).

$$
\sum_{n=1}^{\infty} z^{p}
$$

$p_{n}$ bezeichnet, wie gewöhnlich; die $n$-te Primzahl. Also ist

Man hat somit

$$
a_{n}=\left\{\begin{array}{l}
1, \text { wenn } n \text { Primzahl ist, } \\
0 \text { in allen anderen Fällen. }
\end{array}\right.
$$

$$
M_{\alpha}(s)=\sum_{p} \frac{\chi_{\alpha}(p)}{p^{s}} \quad(\alpha=0,1, \ldots, k-2),
$$

wo die Summation über alle Primzahlen $p$ zu erstrecken ist. Ferner

$$
A_{0}(s)=\frac{1}{k^{8}}
$$

7) M. Fekete, Sur les séries de Dirichlet [Comptes rendus hebdomadaires des séances de l'Académie des Sciences, 150 (1910), S. 1083-1036?; G. H. Hardy, The application to Dirichlet's series of Borel's exponential method of summation [Proceedings of the London Mathematical Society (2), 8 (1910), S. 277-294]. Ubrigens ist der fragliche Satz auch der Arbeit von Hurwitz, Uber die Anwendung eimes funktionentheoretischen Prinzips auf bestimmte Integrale, Math. Annalen, 58, S. 220-224 leicht zu entnehmen. 
Diese Funktionen sind regulär für $\sigma>1$. Es sei nun $s$ reill und $>1$. Die Reihe

$$
\frac{\sum}{p} \frac{\chi_{\alpha}(p)}{p}=\lim _{s=1+0} \sum \frac{\chi_{a}(p)}{p^{s}}
$$

Ist bekanntlich konvergent für $1 \leqq \alpha \leqq k-2$ und eigentlich divergent für $\left.a=0^{8}\right)$. Ferner ist $x_{0} \neq 0$, also nach (5)

$$
\lim _{s=1+0} g(s)=\infty
$$

womit, auf Grund der Ausführungen im $\$ 3$, die Behauptung bewiesen ist, d. h. daß die Reihe in der Uberschrift dièses Paragraphen den Einheitshreis zur natürlichen Grenze hat.

Ähnlich können die Reihen behandelt werden

$$
\begin{gathered}
\sum_{m=1}^{\infty} \sum_{n=1}^{\infty} z^{p_{m} p_{n}}, \sum_{m=1}^{\infty} \sum_{n=1}^{\infty} \sum_{r=1}^{\infty} z^{p_{m} p_{n} p_{r}}, \text { usw. } \\
\S 5 . \\
\sum_{n=1}^{\infty} A(n) z^{n} .
\end{gathered}
$$

$\alpha(n)$ bezeichnet hier das Mangoldtsche Symbol

Man hat

$$
A(n)=\left\{\begin{array}{l}
\log p \text { für eine Primzahlpotenz } n=p^{m}, \\
0 \text { in allen anderen Fällen. }
\end{array}\right.
$$

und

$$
\left.M_{a}(s)=\sum_{n=1}^{\infty} \frac{z_{a}(n) A(n)}{n^{s}}=-\frac{L_{u}^{\prime}(s)}{L_{a}(s)} \quad(c=0,1, \ldots k-2)^{n}\right)
$$

$$
A_{0}(s)=\sum_{n \equiv 0(\bmod k)} \frac{1(n)}{n^{s}}=\sum_{k=1}^{\infty} \frac{1\left(k^{m}\right)}{k^{m} s}=\log k \sum_{m=1}^{\infty} \frac{1}{k^{m s}}=\frac{\log k}{k^{s}-1},
$$

also, nach $\{5\}$,

$$
g(s)=\frac{\log k}{k^{s}-1}-\sum_{a=0}^{k-z} x_{\alpha} \frac{L_{a}^{\prime}(s)}{L_{a}(s)} .
$$

Es sei nun $s=1 ; L_{a}(s)$ ist hier regulär und von 0 versehieden für $\left.1 \leqq c \leqq k-2^{10}\right)$. Die Funktion $L_{0}(s)$ hat hier aber einen Pol erster Ordnung, ähnliches gilt also für ihre logarithmische Ableitang. Ferner ist $x_{0}+0$; also ist $g(s)$ singulär für $s=1$. Darans folgt die Behauptung-

9) H., 1, S. 446.

9) H., 1, 420 .

10) Man vgl. $\$ 1$. 


$$
\sum_{n=1}^{\infty} \sigma(n) z^{n} \text { und } \sum_{n=1}^{\infty} \tau(n) z^{n}
$$

Ich bezeichne mit $\sigma(n)$ die Summe der Teiler von $n$ und mit $\tau(n)$ die Anzahl derselben. Ich setze allgemein

$$
\sigma_{a}(n)=\sum_{d / n} d^{a}
$$

wo $a$ eine beliebige (reelle oder komplexe) Zahl ist ${ }^{11}$ ). Man hat

$$
\sigma_{1}(n)=\sigma(n), \quad \sigma_{0}(n)=\tau(n) .
$$

Ich beweise, daß die Reibe

$$
\sum_{n=1}^{\infty} \sigma_{a}(n) z^{n^{n}}
$$

für sämtliche $a$, welche nicht Warzeln der Gleichung

$$
\zeta(1-a)=0
$$

sind, über den Einheitskreis hinaus nicht fortsetzbar ist. Ist z. B. $a=1$ oder 0 , so ist diese Bedingung erfüllt.

Zunächst ist es klar, daß diese Potenzreihe den Konvergenzradius 1 hat. Es ist nämlich

und für Primzahlen

$$
\sigma_{a}(n) \mid \leqq n \operatorname{Max}\left(1,\left|n^{a}\right|\right)
$$

Ich behaupte nun, daß

$$
\sigma_{a}(p)=1+p^{a} .
$$

$$
M_{\alpha}(s)=L_{\alpha}(s-a) L_{\alpha}(s) \quad(\alpha=0,1, \ldots, k-2)
$$

ist. Wenn ich nämlich das Dirichletsche Produkt der beiden Reihen

bilde, so ist

$$
L_{a}(s-a)=\sum_{n=1}^{\infty} \frac{\chi_{\alpha}(n)}{n^{s-a}}=\sum_{n=1}^{\infty} \frac{\chi_{\alpha}(n) n^{a}}{n^{s}}, \quad L_{\alpha c}(s)=\sum_{n=1}^{\infty} \frac{\chi_{\alpha}(n)}{n^{\delta}}
$$

$$
\sum_{d n} d^{a} \chi_{\alpha}(d) \chi_{\alpha}\left(\frac{n}{d}\right)=\chi_{u}(n) \sum_{d / n} d^{a}=\chi_{\alpha}(n) \sigma_{a}(n) .
$$

Ferner folgt ähnlicherweise

d. h. nach $\left(5^{\prime}\right)$

$$
A(s)=\zeta(s-a) \zeta(s)
$$

$g(s)=\zeta(s-a) \zeta(s)+\left(x_{0}-1\right) L_{0}(s-a) L_{0}(s)+\sum_{a=1}^{k-2} x_{a} L_{a}(s-a) L_{a}(s)$.

i1 $\sum_{d^{\prime} ; n}$ bedeatet, wie gewöhnlich, dab über alle Teiler $d$ von $n$ summiert wird. 
Ist $1 \leqq \alpha \leqq k-2$, so ist $L_{a}(s)$ in der ganzen $s$-Ebene regulär. In der Umgebung der Stelle $s=1$ gilt die Entwickelung

ferner hat man

$$
\zeta(s)=\frac{1}{s-1}+\ldots,
$$

also ist

$$
L_{0}(s)=\left(1-\frac{1}{k^{s}}\right) \zeta(s)
$$

$$
\begin{aligned}
\lim _{s=1}(s-1) g(s) & =\zeta(1-a)\left[1+\left(x_{0}-1\right)\left(1-\frac{1}{k^{1-a}}\right)\left(1-\frac{1}{k}\right)\right] \\
& =\frac{\zeta(1-a)}{k^{1-a}} \text { für } a \neq 0
\end{aligned}
$$

(vgl. (4)) and

$$
\lim _{s=1}(s-1)^{2} g(s)=1+\left(\dot{x}_{0}-1\right)\left(1-\frac{1}{k}\right)^{2}=\frac{1}{k} \quad \text { für } \quad a=0 .
$$

Daraus folgt die Behauptung.

$$
\begin{gathered}
\S 7 . \\
\sum_{n=1}^{\infty} \varphi(n) z^{n} .
\end{gathered}
$$

$\varphi(n)$ bezeichnet hier das Eulersche Zeichen: die Anzahl der Zahlen $x<n$, die zu $n$ teilerfremd sind. Es ist offenbar $p(n)<n$ und für Primzahlen $\varphi(p)=p-1$. Die Patenzreihe

$$
\sum_{n=1}^{\infty} p(n) z^{n}
$$

hat somit den Konvergenzradius 1.

Man hat

$$
M_{\alpha}(s)=\frac{L_{\alpha}(s-1)}{L_{\alpha}(s)} \quad(c=0,1, \ldots, k-2)
$$

und

Also ist nach $\left(5^{\prime}\right)$

$$
\left.A(s)=\frac{\zeta(s-1)}{\zeta(s)}{ }^{12}\right)
$$

$$
g(s)=\frac{\zeta(s-1)}{\zeta(s)}+\left(x_{0}-1\right) \frac{L_{0}(s-1)}{L_{s}(s)}+\sum_{s=1}^{n-z} x_{\alpha} \frac{L_{\alpha}(s-1)}{L_{\text {es }}(s)} .
$$

Es sei nun $s=2$. $M_{\alpha}(s)$ ist regulär für $1 \leqq c \leqq k-2$; das Residuum ist somit

q. e. d.

$$
\lim _{s=2}(s-2) g(s)=\frac{1}{b(2)}\left[1+\left(x_{0}-1\right) \frac{1-\frac{1}{k}}{1-\frac{1}{k^{2}}}\right]=\frac{1}{\zeta(2)} \frac{1}{i-k^{2}}+0,
$$

18) Man vgl. H., 2, S. 680 . 


$$
\sum_{n=1}^{\infty} 2^{v(n)} z^{n}
$$

$\nu(n)$ bezeichnet hier die Anzahl der verschiedenen Primfaktoren in $n[v(1)=0]$. Es ist offenbar $1 \leqq 2^{v(n)} \leqq \tau(n)$; ist nämlich

so sind die Zahlen

$$
n=p_{1}^{\delta_{1}} p_{2}^{\delta_{2}} \cdots p_{v}^{\delta_{v}}
$$

$$
[\nu=v(n)]
$$

$$
1 ; p_{1}, p_{2}, p_{3}, \ldots ; p_{1} p_{2}, p_{1} p_{3}, \ldots ; p_{1} p_{2} p_{3}, \ldots \text {, }
$$

deren Anzahl eben $2^{v(\boldsymbol{n})}$ ist, alle Teiler von $n$. Die Potenzreihe

$$
\sum_{n=1}^{\infty} 2^{\nu(n)} z^{n}
$$

hat somit den Konvergenzradius 1 .

Man hat

Nun ist

$$
\left.M_{a}(s)=\left[L_{\alpha}(s)\right]^{2} \prod_{p}\left[1-\frac{z_{\alpha}\left(p^{2}\right)}{p^{2 s}}\right]^{13}\right)
$$

d. h.

$$
\chi_{\alpha \alpha}\left(p^{2}\right)=\left[\chi_{\alpha}(p)\right]^{2}=\chi_{2 \alpha}(p)
$$

and ähnlicherweise

$$
M_{\alpha}(s)=\frac{\left[L_{\alpha}(s)\right]^{2}}{L_{2 \alpha}(2 s)} \quad(\alpha=0,1, \ldots, k-2)
$$

$$
A(s)=\frac{[\zeta(s)]^{2}}{\zeta(2 s)} .
$$

Es sei nun $s=1 . \quad M_{\alpha}(s)$ ist regulär für $1 \leqq \alpha \leqq k-2 ; g(s)$ hat. den zweifachen Pol $s=1$, denn es ist nach den Formeln $\left(5^{\prime}\right)$ und (4) im $\S 2$

$$
\lim _{s=1}(s-1)^{2} g(s)=\frac{1}{\zeta(2)}\left[1+\left(x_{0}-1\right) \frac{\left(1-\frac{1}{k}\right)^{2}}{1-\frac{1}{k^{2}}}\right]=\frac{1}{\zeta(2)} \frac{1}{1+k} \neq 0,
$$

q. e. d.

$$
\S 9 .
$$

$$
\sum_{n=1}^{\infty} \mu(n) z^{n}
$$

$\mu(n)$ bezeichnet das Möbiussche Symbol:

$\mu(1)=1$,

$\mu(n)=0$ für nicht quadratfreie $n$,

13) H., 2, S. 682 . 
Poteuzreihen, deren Koeffizienten zahlentheoretische Funktionen sind.

$\mu(n)=(-1)^{r}$, wenn $n$ quadratfrei ist und aus $r$ verschiedenen Primfaktoren besteht.

Man hat

$$
M_{\alpha}(s)=\sum_{n=1}^{\infty} \frac{x_{a}(n) \mu(n)}{n^{s}}=\frac{1}{L_{\alpha}(s)} \quad(r=0,1, \ldots, k-2)
$$

und

$$
\left.A(s)=\sum_{n=1}^{\infty} \frac{\mu(n)}{n^{8}}=\frac{1}{\xi(s)}{ }^{14}\right) \text {. }
$$

Also ist

$$
g(s)=\frac{1}{\zeta(s)}+\frac{x_{0}-1}{L_{0}(s)}+\sum_{\alpha=1}^{k-2} \frac{x_{\alpha}}{L_{\alpha}(s)} .
$$

Ich betrachte diese Funktion an den Stellen $s=-1,-3,-5, \ldots$ Hier verschwinden nur die $L_{a}(s)$ mit ungeradem $a^{15}$ ); ich setze

$$
R_{q}=\lim _{q=-(2 q-1)} g(s)(s+2 q-1) \quad(q=1,2,3, \ldots
$$

Man bedenke ferner, daß für ungerade $\alpha$

$$
L_{c}(s)=\left(\frac{\pi}{k}\right)^{\frac{s+1}{2}} \frac{1}{\Gamma\left(\frac{s+1}{2}\right)} \xi_{a}(s)
$$

ist und

$$
\lim _{s=-m}(s+m) \Gamma(s)=\frac{(-1)^{m}}{m !} \quad(m=0,1,2, \ldots)
$$

Man hat somit

$$
\begin{aligned}
\lim _{s=-\{2 q-1\}} \frac{L_{\alpha}(s)}{s+2 q-1} & =A_{q} \xi_{\alpha}(-2 q+1)=A_{q} \varepsilon_{\alpha} \xi_{k-1-\alpha}(2 q) \\
& =B_{q} \varepsilon_{\alpha} L_{k-1-\alpha}(2 q)=B_{q} \varepsilon_{\alpha} \sum_{n=1}^{\infty} \frac{\bar{z}_{\alpha q}(n)}{n^{2 q}}
\end{aligned}
$$

wo $A_{q}$ und $B_{q}$ von 0 verschieden sind und außer $q$ nur von $k$ abhängenNun ist

also

$$
\sum_{n=1}^{\infty} \frac{\bar{x}_{\alpha}(n)}{n^{2 q}} \sum_{n=1}^{\infty} \frac{\bar{x}_{\alpha}(n) \mu(n)}{n^{2 q}}=1
$$

$$
\lim _{=-(2 q-1)} \frac{s+2 q-1}{\mathcal{L}_{a}(s)}=\frac{1}{\bar{B}_{q} \varepsilon_{a}} \sum_{n=1}^{\infty} \frac{\bar{\gamma}_{a}(n) \mu(n)}{n^{2 q}} ;
$$

14) Man vgl. H., 2, S.576.

15) Man vgl. \& $1 ; \frac{1}{\Gamma(s)}$ hat nämlich die Wurzeln $s=0,-1,-2, \ldots$ 
daraus folgt, wenn ich

setze, daß

$$
d_{n}=\frac{x_{1}}{\varepsilon_{1}} \bar{\chi}_{1}(n)+\frac{x_{3}}{\varepsilon_{3}} \bar{\chi}_{3}(n)+\ldots+\frac{x_{k-2}}{\varepsilon_{k-2}} \bar{\chi}_{k-2}(n)
$$

$$
R_{q}=\frac{1}{B_{q}} \sum_{n=1}^{\infty} \frac{d_{n} \mu(n)}{n^{2 q}} \quad(q=1,2,3, \ldots)
$$

ist. Wäre nun $R_{q}=0$ für alle $q$, so hätte die Dirichletsche Reihe

$$
\sum_{n=1}^{\infty} \frac{d_{n} \mu(n)}{n^{s}}
$$

Wurzeln mit beliebig großer Abszisse.

Es gilt aber der Satz:

Hat die Dirichletsche Reihe einen absoluten Konvergenzbereich und Wurzeln mit beliebig großer Abszisse, so verschwindet sie identisch ${ }^{16}$ ).

In diesem Falle ist

also

$$
\left|d_{n} \mu(n)\right| \leqq\left|x_{1}\right|+\left|x_{3}\right|+\cdots+x_{k-2} \mid=O(1),
$$

$$
d_{n} \mu(n)=0 \quad(n=1,2,3, \ldots) .
$$

Es ist aber $d_{n}=d_{n^{\prime}}$ für $n \equiv n^{\prime}(\bmod k)$; ist ferner $1 \leqq r \leqq k-1$, so enthält die arithmetische Progression $r+k x$ immer eine Primzahl (sogar unendlich viele Primzahlen), für welche also

$$
\mu(n)=\mu(r+k x)=-1 \neq 0
$$

ist. Also ist $d_{n}=d_{r}=0$ für $r=1,2, \ldots k-1$, d. h.

$$
\frac{x_{1}}{\varepsilon_{1}} \bar{\chi}_{1}(n)+\frac{x_{3}}{\varepsilon_{3}} \bar{\chi}_{3}(n)+\ldots+\frac{x_{k-2}}{\varepsilon_{k-2}} \bar{\chi}_{k-2}(n)=0 \quad(n=1,2, \ldots, k-1) .
$$

Es ist also insbesondere

$$
\frac{x_{1}}{\delta_{1}} \bar{\varphi}^{v}+\frac{x_{3}}{\varepsilon_{3}} \bar{\rho}^{3 v}+\ldots+\frac{x_{k-2}}{\varepsilon_{k-2}} \bar{\varphi}^{(k-2) \nu}=0 \quad\left(0 \leqq \nu \leqq \frac{k-3}{2}\right) .
$$

Nun ist $\left|\varepsilon_{\alpha}\right|=1$, und die Zahlen $x_{1}, x_{3}, \ldots, x_{k-2}$ können nicht sämtlich verschwinden (vgl. §2); also ist die Determinante dieses Systems gleich 0. Dies trifft aber nicht $\mathrm{zu}$, da $\bar{\varphi}^{\alpha} \neq \bar{\varphi}^{\beta}$ für $2 \leqq|\alpha-\beta| \leqq k-2$. Damit ist die Behauptung bewiesen.

16) H., 2, S. 744, Satz 15. 
Man hat

$$
\sum_{n=1}^{\infty}[\mu(n)]^{2} z^{n}
$$

$$
\begin{array}{r}
H_{a}(s)=\sum_{n=1}^{\infty} \frac{\chi_{\alpha}(n)[\mu(n)]^{2}}{n^{s}}=\prod_{p}\left[1+\frac{\chi_{\alpha}(p)}{p^{s}}\right]=\prod_{p}^{1-\frac{\chi_{2 \alpha}(p)}{p^{28}}} \frac{L_{\alpha a}(s)}{1-\frac{\chi_{\alpha}(p)}{p^{8}}}=\frac{L_{a s}(2 s)}{(k-0,1, \ldots, k-2\}}
\end{array}
$$

und

Also ist

$$
A(s)=\frac{5(s)}{\zeta(2 s)}
$$

$$
g(s)=\frac{\zeta(s)}{\zeta(2 s)}+\left(x_{0}-1\right) \frac{L_{0}(s)}{L_{0}(2 s)}+\sum_{\alpha=1}^{k-2} x_{\alpha} \frac{L_{\alpha}(s)}{L_{\alpha}(2 s)} .
$$

Es sei $s=1$; das Residuum ist

$$
\lim _{s=1}(s-1) g(s)=\frac{1}{\zeta(2)}+\left(x_{0}-1\right) \frac{1-\frac{1}{k}}{1-\frac{1}{k^{2}}} \frac{1}{\zeta(2)}=\frac{1}{\zeta(2)} \frac{1}{1-k^{2}}+0
$$

q. e.d.

Hier ist

$$
\sum_{n=1}^{\infty} 2^{\gamma(n)} \lambda(n) z^{n}
$$

$\lambda(1)=1$

$\lambda(n)=(-1)^{r}$, wemn die Anzahl der Primfaktoren in $n$ (mehriache mit ihrer Multiplizität gezählt) $r$ ist.

Man hat

und

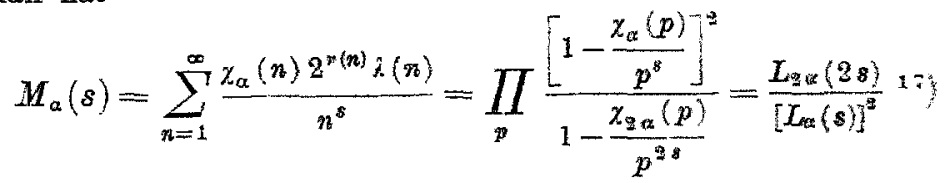

$$
\begin{aligned}
& (a=0,1, \ldots, k-2)
\end{aligned}
$$

Also ist

$$
A(s)=\frac{5(2 s)}{[5(s)]^{2}}
$$

$$
g(s)=\frac{\zeta(2 s)}{[\zeta(s)]^{2}}+\left(x_{0}-1\right) \frac{L_{b}(28)}{\left[L_{b}(s)\right]^{2}}+\sum_{a=1}^{k-2} x_{n a} \frac{L_{s} a(2 s)}{\left[L_{n}(s)\right]^{2}}
$$

17) H., 2, S. 678 .

3thematiache Zeitgchrift. $\mathrm{VII}$. 
Ich betrachte diese Funktion an den Stellen $s=-1,-3,-5, \ldots$, Ich setze wie in $\$ 9$

$$
R_{q}=\lim _{s=-(2 q-1)} g(s)(s+2 q-1) \quad(q=1,2,3, \ldots) .
$$

Dann ist, wie dort, für ungerade $\alpha$

$$
\lim _{s=-(2 q-1)} \frac{s+2 q-1}{L_{n}(s)}=\frac{1}{B_{q} \varepsilon_{\dot{\alpha}}} \sum_{n=1}^{\infty} \frac{\bar{z}_{\alpha}(n) \mu(n)}{n^{2 q}}
$$

ferner ist ähnlicherweise

$$
\lim _{s=-(2 q-1)} \frac{L_{2 \alpha}(2 s)}{s+2 q-1}=C_{q} \varepsilon_{2 \alpha} \sum_{n=1}^{\infty} \frac{\bar{z}_{2 \alpha}(n)}{n^{4 q-1}},
$$

wo $C_{q}$ von 0 verschieden ist und außer $q$ nur von $k$ abhängt.

Daraus folgt für ungerade $a$

$$
\lim _{s=-(2 q-1)}(s+2 q-1) \frac{L_{2 \alpha}(2 s)}{\left[L_{u(s}(s)\right]^{2}}=\frac{C_{q}}{B_{q}^{\tilde{s}}} \frac{\varepsilon_{3 \alpha}}{\varepsilon_{\alpha}^{2}}\left\{\sum_{n=1}^{\infty} \frac{\bar{x}_{\alpha}(n) \mu(n)}{n^{2 q}}\right\}^{2} \sum_{n=1}^{\infty} \frac{\bar{z}_{2 \alpha}(n)}{n^{4 q-1}} .
$$

Ich definiere nun die folgenden zahlentheoretischen Funktionen:

Ferner sei

$$
\begin{aligned}
& G(n)=0, \text { wenn } n \text { keine Quadratzahl ist, } \\
& G(n)=\nu \text {, wenn } n=\nu^{2} \text { ist. }
\end{aligned}
$$

und

$$
\mu^{*}(n)=\sum_{d / n} \mu(d) \mu\left(\frac{n}{d}\right)
$$

Dann ist offenbar

$$
F(n)=\sum_{d / n} \mu^{*}(d) G\left(\frac{n}{d}\right)
$$

und

also

$$
\left\{\sum_{n=1}^{\infty} \frac{\bar{x}_{a}(n) \mu(n)}{n^{s}}\right\}^{2}=\sum_{n=1}^{\infty} \frac{\bar{x}_{a}(n) \mu^{*}(n)}{n^{s}}
$$

$$
\sum_{n=1}^{\infty} \frac{\tilde{\chi}_{9 \alpha}(n)}{n^{2 s-1}}=\sum_{n=1}^{\infty} \frac{\bar{\chi}_{\alpha}\left(n^{2}\right) n}{n^{2 s}}=\sum_{n=1}^{\infty} \frac{\bar{\chi}_{\alpha}(n) G(n)}{n^{s}}
$$

$$
\left\{\sum_{n=1}^{\infty} \frac{\bar{z}_{n \alpha}(n) \mu(n)}{n^{s}}\right\}^{2} \sum_{n=1}^{\infty} \frac{\bar{y}_{2 a}(n)}{n^{28-1}}=\sum_{n=1}^{\infty} \frac{\bar{z}_{\alpha}(n) F^{r}(n)}{n^{s}} .
$$

Ich setze

dann ist

$$
e_{n}=x_{1} \frac{\varepsilon_{9}^{3}}{\varepsilon_{1}^{3}} \bar{\chi}_{1}(n)+x_{3} \frac{\varepsilon_{B}^{3}}{\varepsilon_{3}^{3}} \bar{\chi}_{3}(n)+\ldots+x_{k-2} \frac{\varepsilon_{2 k-4}}{\varepsilon_{k-2}^{2}} \bar{\chi}_{k-2}(n),
$$

$$
R_{q}=\frac{C_{q}}{B_{q}^{q}} \sum_{n=1}^{\infty} \frac{e_{n} F(n)}{n^{3} q} \quad(q \geqq 1 \text { und } \operatorname{ganz})
$$


Wäre also $R_{q}=0$ für $q=1,2,3, \ldots$, so hätte die Dirichletsche Reihe

$$
\sum_{n=1}^{\infty} \frac{e_{n} F(n)}{n^{s}}
$$

Wurzeln mit beliebig großer Abszisse. Nun ist

man hat ferner

$$
e_{n}=O(1)
$$

und

$$
\mu^{*}(n) \sum^{*} \leqq \sum_{d ; n} 1 \leqq n
$$

Daraus folgt nach $\$ 9$

$$
F(n) \leqq \sum_{d / n} d \sqrt{\frac{n}{d}} \leqq n \sqrt{n} \sqrt{n}=n^{2} .
$$

$$
e_{n} F(n)=0
$$

$n=1,2,3, \ldots)$

Man hat aber wie dort $e_{n}=e_{n^{\prime}}$ für $n=n^{\prime}(\bmod k)$; ferner ist, wenn $p$ eine Primzahl bezeichnet,

also

$$
\mu^{*}(1)=1, \quad \mu^{*}(p)=2 \mu(p)=-2,
$$

D. h.

$$
F(p)=G(p)-2 G(1)=-2+0 \text {. }
$$$$
e_{1}=e_{2}=\ldots=e_{k-1}=0 \text {. }
$$

Daraus folgt ebenso wie in $\S 9$ die Unmöghichkeit der Annohme, dab $i ; s)$ eine ganze transzendente Funktion ist. Damit ist die Behauptung bewiesen.

(Eingegangen am 4. August 1919.) 